\title{
USO DE GABAPENTINA NO TRATAMENTO DA SÍNDROME SUNCT
}

\author{
Pedro Augusto Sampaio Rocha Filho', Getulio D. Rabello', \\ Antonio C. Ribeiro Galvão', Ida Fortini', Marcelo Calderaro', Dalva Carrocini ${ }^{2}$
}

\begin{abstract}
RESUMO - Relatamos o caso clínico de duas mulheres com quadro compatível com síndrome SUNCT (cefaléia de curta duração, unilateral, neuralgiforme com hiperemia conjuntival e lacrimejamento). As duas apresentavam exames clínico e neurológico normais e RM com sinais de microangiopatia. A primeira apresentava cefaléia há três anos, que ocorria várias vezes por dia, sempre que mastigava ou bocejava. Havia feito uso várias medicações sem melhora. A dor foi controlada após o uso de $600 \mathrm{mg}$ de gabapentina ao dia. A segunda paciente referia cefaléia há seis meses. A dor era diária, ocorrendo de 20-40 vezes por dia. $\mathrm{Na}$ ocasião da primeira avaliação no ambulatório, já fazia uso $600 \mathrm{mg}$ de carbamazepina ao dia e $15 \mathrm{mg}$ de clorpromazina, com melhora parcial. Após introdução de gabapentina- 1200 mg/ dia, a paciente evoluiu sem dor, porém com episódios de hiperemia conjuntival.
\end{abstract}

PALAVRAS-CHAVE: SUNCT, cefaléia, tratamento, gabapentina, dor, cefaléia ultra-curta.

\begin{abstract}
Gabapentin in the treatment of SUNCT syndrome
ABSTRACT - We report the cases of two women who presented a clinical condition compatible with the SUNCT (short-lasting unilateral neuralgiform headache attacks with conjunctival injection and tearing) syndrome. Both presented normal clinical and neurological examination and MRI compatible with microangiopathy. The first one related headache attacks for three years, occurring several times a day when she masticated or yawned. She had a history of multiple failed therapies. The pain was controlled after the use of gabapetin ( $600 \mathrm{mg}$ per day). The second one related she had daily headaches for six months that occurred from 20 to 40 times per day. At the first visit to the ambulatory, she related she was using carbamazepine (600 mg per day) and chlorpromazine (15 mg per day) having a partial response. Administration of gabapentin (1200 mg per day) led to complete resolution of the pain attacks, but the patient continue to have episodes of conjunctival injection.
\end{abstract}

KEY WORDS: SUNCT, headache, treatment, gabapentin, pain, ultra-shorting headaches.

A síndrome SUNCT (short-lasting unilateral neuralgiform headache attacks with conjuntival injection and tearing) foi descrita pela primeira vez por Sjaastad e colaboradores em 1989', só recentemente sendo incluída na segunda edição da Classificação Internacional de Cefaléias no grupo que inclui a cefaléia em salvas e outras cefaléias trigêmino-autonômicas². Esta cefaléia é caracterizada por dor unilateral, orbitária, supra-orbitária ou temporal, em pontada ou pulsátil, com duração de 5-240 segundos, associada a hiperemia conjuntival e lacrimejamento ipsilaterais a mesma ${ }^{2}$. Os sintomas autonômicos, em geral dramáticos, iniciam-se rapidamente após o início da dor, podendo haver além dos já citados, rinorréia e obstrução nasal ${ }^{1,3,4}$. As crises podem ter freqüência variável, de uma a duas crises por dia a até 10-30 crises por hora, de ocorrência geralmente diurna ${ }^{5}$, podendo ser precipitada por manobras mecânicas do pescoço e por zonas gatilho em topografias trigeminais e extra-trigeminais, geralmente não se observando períodos refratários após estímulos sucessivos ${ }^{1,4,6}$.

Esta síndrome inicia-se geralmente entre os $41 \mathrm{e}$ 70 anos (média de 51 anos) ${ }^{4}$ e tem uma predominância do sexo masculino $0^{4,5}$. As crises têm padrão irregular, com alternância errática entre períodos com dor, que variam de dias a meses e geralmente ocorrem 1 a 2 vezes por ano, e períodos de remissão que podem durar meses ${ }^{4}$.

Dentre as cefaléias primárias, temos como diagnósticos diferenciais a hemicrânia paroxística crônica, a cefaléia em salvas, a neuralgia do primeiro ramo do trigêmio e a cefaléia idiopática em facadas ${ }^{7}$. Apesar

Ambulatório de Cefaléias da Clínica Neurológica do Hospital das Clínicas da Universidade de São Paulo, São Paulo SP, Brasil: ${ }^{1}$ Neurologista; ${ }^{2}$ Psiquiatra.

Recebido 3 Outubro 2006, recebido na forma final 7 Dezembro 2006. Aceito 22 Fevereiro 2007. 
da maioria dos casos descritos serem primários, casos secundários foram descritos ${ }^{8}$, devendo todos os casos serem investigados com exames de imagem. Uma característica proeminente desta síndrome é a irresponsividade ao tratamento clínico ${ }^{3,9}$. Há relatos de melhora com o uso de carbamazepina, amitriptilina, predinisona, topiramato ${ }^{7}$, nefedipina, sumatripitano $^{9}$, lamotrigina ${ }^{10}$, oxicarbamazepina ${ }^{11}$ e gabapentina ${ }^{12-15}$. Bloqueios anestésicos não funcionaram, havendo relato de melhora após bloqueio local do gânglio cervical superior com opióide ${ }^{16}$. Existem relatos de procedimentos cirúrgicos que tiveram êxito ${ }^{7}$ e também de respostas negativas ${ }^{17}$.

Descreveremos duas pacientes tratadas com êxito com gabapentina.

\section{CASOS}

Caso 1 - Mulher de 75 anos apresentando, há três anos, dor em fisgada em região frontal e periorbitária esquerda, de grande intensidade e duração de 2-3 minutos. A dor é acompanhada de hiperemia conjuntival e lacrimejamento e é desencadeada pela mastigação, bocejo, falar e sorrir, não se verificando tempo de latência. Inicialmente a dor ocorria com freqüência de 2-3 vezes por semana, aumentando sua freqüência gradativamente até tornar-se diária após um ano. Após um ano e meio do início do quadro, a paciente passou a ter em torno de 50 crises por dia, sempre que mastigava ou bocejava. Ficou com esta freqüência por seis meses. Refere também crises noturnas, entretanto estas ocorriam em menor freqüência (4-5x /noite), porém diariamente e chegavam a acordar a paciente. A paciente havia feito uso de carbamazepina (400 mg/dia por uma semana); indometacina (50 mg/dia por um mês), clonazepam ( $2 \mathrm{mg}$ 12/12 horas por um mês), amitriptilina (25 mg por dia por uma semana) e acupuntura sem melhora. Apresentava exames clínico e neurológico normais.

Durante a investigação foram feitos USG Doppler de carótidas, EEG, VHS, glicemia de jejum, função renal e hepáticas, com resultados normais. A RM apresentava sinais de microangiopatia.

Iniciou-se gabapentina (300 mg 2x/dia), com melhora da freqüência de crises. Após três semanas do início da medicação, já apresentou melhora da freqüência (2-3x/dia), permanecendo com freqüência de uma vez por dia por cinco meses, quando ficou sem dor. Ficou sem dor após 6 meses. Atualmente sem dor com gabapentina, $600 \mathrm{mg} / \mathrm{dia}$, há seis meses. Durante todo período de acompanhamento no nosso ambulatório, fez uso exclusivamente de gabapentina e não apresentou efeitos colaterais relacionados à droga.

Caso 2 - Mulher de 82 anos, com cefaléia em pontadas, de forte intensidade, retro-orbitária direita, com duração de até 30 segundos, há seis meses. A dor era diária, ocorrendo com freqüência de 20-40 vezes por dia e acompanhada de lacrimejamento e hiperemia conjuntival ipsilaterais. A crise pode ser desencadeada por estímulos sensoriais táteis em hemiface direita como, por exemplo, passar um al- godão sobre essa hemiface. À admissão no ambulatório, já fazia uso carbamazepina - $600 \mathrm{mg} /$ dia e clorpromazina $4 \%$ - 15 mg/dia, com melhora parcial. Apresentava exame clínico e neurológico normais. Realizados CT de crânio e LCR normais. A RM mostrou sinais de microangiopatia. Optouse por retirar a carbamazepina e a clorpromazina devido à idade da paciente. Introduziu-se gabapentina - $400 \mathrm{mg} / \mathrm{dia}$ que foi aumentada gradativamente a cada cinco dias até a dose de $400 \mathrm{mg}$ de $8 / 8$ horas. A paciente evoluiu sem dor com o uso exclusivo deste medicamento, porém com episódios de hiperemia conjuntival. Não houve efeitos colaterais relacionados ao uso da gabapentina.

\section{DISCUSSÃO}

As cefaléias trigêmino-autonômicas têm em comum a ativação entre a aferência trigeminal (responsável pela dor) e eferência parassimpática do nervo facial (responsável pelos sintomas autonômicos), integradas no tronco encefálico ${ }^{18}$. No SUNCT foi descrita uma ativação da área postero-inferior do hipotálamo ipsilateral a dor ${ }^{19}$, mesma alteração encontrada na cefaléia em salvas ${ }^{20}$, bem como uma ativação hipotalâmica bilateral ${ }^{21}$. Esses achados falam a favor de uma origem central para esta cefaléia. A síndrome SUNCT tem importantes semelhanças com a neuralgia do primeiro ramo do nervo trigêmeo (V1), seu principal diagnóstico diferencial. Encontramos em ambos a mesma localização, mesmo caráter da dor, presença de pontos gatilhos, ocorrência na mesma faixa etária.

A neuralgia de $\mathrm{V} 1$ é entidade nosológica rara, de ocorrência dos 26 aos 81 (média 56,2 anos), com a dor durando em geral "segundos", podendo chegar a 30 segundos ou mais e ocorrer à noite e nesse caso, podendo acordar o paciente. Geralmente responde bem ao tratamento com carbamazepina e, ao contrário do SUNCT, tem períodos refratários quando realizados estímulos repetitivos. Verifica-se uma alternância errática entre períodos sintomáticos e assintomáticos e a dor, com o tempo, tende a estenderse para território de $\mathrm{V} 2$ e $\mathrm{V}^{22}$. A grande diferença entre as duas entidades é a presença de sintomas autonômicos marcantes no SUNCT. Na neuralgia do trigêmio podemos encontrar também sintomas autonômicos ${ }^{22-25}$ como hiperemia conjuntival e lacrimejamento, porém, menos pronunciados ${ }^{24}$.

No segundo caso descrito, a paciente continuou a apresentar episódios de hiperemia conjuntival sem dor. A presença de sintomas autonômicos sem dor é rara no SUNCT ${ }^{1,26}$ sugerindo uma ativação parassimpática mesmo fora do ataque. Até onde temos conhecimento, a permanência de ataques paroxísticos de hiperemia conjuntival após a dor ter sido controlada não havia ainda sido descrita. 
Se aceitarmos que a ativação dolorosa de V1 resulta na ativação parassimpática de algum nível, em geral subclínica, devemos então esperar estes sintomas autonômicos na neuralgia de $\mathrm{V}^{27}$. É interessante salientar que existem descrições de neuralgia trigeminal que evoluiu para SUNCT ${ }^{28}$ ou que nos períodos de dor mais intensa, apresentava-se fenotipicamente Como SUNCT ${ }^{29}$.

Os casos relatados neste artigo apresentaram boa resposta ao uso de gabapentina. A gabapentina é um análogo estrutural do ácido gama-aminobutírico (GABA). Não atua nos receptores gabaérgicos, não inibe a recaptação nem a degradação do GABA. Seu mecanismo de ação não esta totalmente esclarecido. Aumenta o GABA e a serotonina e diminui o glutamato no SNC, o que lhe confere eficácia no tratamento das dores neuropáticas. Provavelmente age bloqueando os canais de cálcio do tipo $L$, subunidade $\alpha_{2} \delta$ e reduzindo discretamente a síntese de glutamato. É discutível sua ação como agonista parcial no local da glicina, modulada no receptor NMDA ${ }^{30}$.

Além da gabapentina, há relatos na literatura de casos que responderam a carbamazepina, amitripti$\operatorname{lina}^{7}$ e lamotrigina ${ }^{10}$, drogas que podem ser usadas igualmente para neuralgia do trigêmeo. Relatam-se também procedimentos cirúrgicos usados para neuralgia trigeminal que trouxeram bons resultados no tratamento do SUNCT ${ }^{7}$.

Consideramos que ainda há espaço para discussão se o SUNCT constitui uma entidade nosológica própria, com fisiopatologia diversa da neuralgia trigeminal ou se faz parte do espectro desta última, representando os casos com dor que tem duração maior, sintomas autonômicos pronunciados e em geral, maior refratariedade às medicações usadas.

O fato de a síndrome SUNCT ser uma cefaléia rara e de suas crises terem padrão irregular, com alternância errática entre períodos com e sem dor, dificulta a avaliação das opções terapêuticas. A boa resposta à gabapentina apresentada por nossas pacientes reforça a inclusão desta medicação entre o arsenal terapêutico disponível para o tratamento da síndrome SUNCT.

\section{REFERÊNCIAS}

1. Sjaastad O, Saunte C, Salvesen R, et al. Shortlasting unilateral neuralgiform headache attacks with conjunctival injection, tearing, sweating, and rhinorrhea. Cephalalgia 1989;9:147-156.

2. The International Classification of Headache Disorders: 2.Ed. Cephalalgia 2004;24(Suppl 1):S9-S160.
3. Pareja J, Caminero A, Sjaastad O. SUNCT syndrome: diagnosis and treatment. Headache 2003;43:306.

4. Pareja JA, Sjaastad O. SUNCT syndrome: a clinical review. Headache 1997;37:195-202.

5. Pareja JA, Shen JM, Kruszewski P, Caballero V, Pamo M, Sjaastad O SUNCT syndrome: duration, frequency, and temporal distribution of attacks. Headache 1996;36:161-165.

6. Lain AH, Caminero AB, Pareja JA. SUNCT syndrome; absence of refractory periods and modulation of attack duration by lengthening of the trigger stimuli. Cephalalgia 2000;20:671-673.

7. Pareja JA, Caminero AB, Sjaastad O. SUNCT syndrome: diagnosis and treatment. CNS Drugs 2002;16:373-383.

8. Rocha PA Filho, Galvao AC, Teixeira MJ, et al. SUNCT syndrome associated with pituitary tumor: case report. Arq Neuropsiquiatr 2006;64: 507-510.

9. Pareja JA, Kruszewski P, Sjaastad O. SUNCT syndrome: trials of drugs and anesthetic blockades. Headache 1995;35:138-142.

10. Piovesan EJ, Siow C, Kowacs PA, Werneck LC. Influence of lamotrigine over the SUNCT syndrome: one patient follow-up for two years. Arq Neuropsiquiatr 2003;61:691-694.

11. Dora B. SUNCT syndrome with dramatic response to oxcarbazepine. Cephalalgia 2006;26:1171-1173.

12. Graff-Radford SB. SUNCT syndrome responsive to gabapentin (Neurontin). Cephalalgia 2000;20:515-517.

13. Hunt $\mathrm{CH}$, Dodick DW, Bosch EP. SUNCT responsive to gabapentin. Headache 2002;42:525-526.

14. Porta-Etessam J, Benito-Leon J, Martinez-Salio A, Berbel A. Gabapentin in the treatment of SUNCT syndrome. Headache 2002;42:523-524.

15. Porta-Etessam J, Martinez-Salio A, Berbel A, Benito-Leon J. Gabapentin (neuronetin) in the treatment of SUNCT syndrome. Cephalalgia 2002; 22: 249; author reply 249-250.

16. Sabatowski R, Huber M, Meuser T, Radbruch L. SUNCT syndrome: a treatment option with local opioid blockade of the superior cervical ganglion? A case report. Cephalalgia 2001;21:154-156.

17. Black DF, Dodick DW. Two cases of medically and surgically intractable SUNCT: a reason for caution and an argument for a central mechanism. Cephalalgia 2002;22:201-204.

18. Goadsby PJ, Lipton RB. A review of paroxysmal hemicranias, SUNCT syndrome and other short-lasting headaches with autonomic feature, including new cases. Brain 1997;120:193-209.

19. May A, Bahra A, Buchel C, Turner R, Goadsby PJ. Functional magnetic resonance imaging in spontaneous attacks of SUNCT: short-lasting neuralgiform headache with conjunctival injection and tearing. Ann Neurol 1999;46:791-794.

20. May A, Bahra A, Buchel C, Frackowiak RS, Goadsby PJ. Hypothalamic activation in cluster headache attacks. Lancet 1998;352:275-278.

21. Sprenger T, Valet M, Platzer S, Pfaffenrath V, Steude U, Tolle TR. SUNCT: bilateral hypothalamic activation during headache attacks and resolving of symptoms after trigeminal decompression. Pain 2005;113 $422-426$.

22. Sjaastad O, Pareja JA, Zukerman E, Jansen J, Kruszewski P. Trigeminal neuralgia: clinical manifestations of first division involvement. Headache 1997;37:346-357.

23. Benoliel R, Sharav Y. Trigeminal neuralgia with lacrimation or SUNCT syndrome? Cephalalgia 1998; 18: 85-90.

24. Pareja JA, Baron M, Gili P, et al. Objective assessment of autonomic signs during triggered first division trigeminal neuralgia. Cephalalgia 2002;22:251-255.

25. Penman J. The differential diagnosis and treatment of tic douloureux. Postgrad Med J 1950;26:627-636.

26. Schwaag S, Frese A, Husstedt IW, Evers S. SUNCT syndrome: the first German case series. Cephalalgia 2003;23:398-400.

27. Goadsby PJ, Matharu MS, Boes CJ. SUNCT syndrome or trigeminal neuralgia with lacrimation. Cephalalgia 2001;21:82-83.

28. Bouhassira D, Attal N, Esteve M, Chauvin M. "SUNCT" syndrome: a case of transformation from trigeminal neuralgia? Cephalalgia 1994 14:168-170.

29. Sesso RM. SUNCT syndrome or trigeminal neuralgia with lacrimation and conjunctival injection? Cephalalgia 2001;21:151-153.

30. Teixeira MJ. Dor: contexto interdisciplinar. Curitiba: Maio, 2003: 1834. 